\title{
Probing Electronic Structures of Monolayer WSe2 Stacked with hBN Using Correlative Cathodoluminescence and Electron Energy-Loss Spectroscopy
}

Wei-Chang Yang ${ }^{1}$, Hsun-Jen Chuang ${ }^{2}$, Matthew Rosenberger ${ }^{3}$, Kathleen McCreary ${ }^{4}$, Berend Jonker ${ }^{4}$ and Renu Sharma 5

${ }^{1}$ National Insitute of Standards and Technology, United States, ${ }^{2}$ Nova Research, Inc., United States, ${ }^{3}$ Department of Aerospace and Mechanical Engineering, University of Notre Dame, United States, ${ }^{4}$ Materials Science \& Technology Division, Naval Research Laboratory, United States, ${ }^{5}$ National Institute of Standards and Technology, United States

Atomically thin 2D transition metal dichalcogenides (TMDC) have attracted research interests for their unique optoelectronic properties that are not displayed in the bulk form [1]. For many device applications, hexagonal boron nitride $(\mathrm{hBN})$ is used to support or protect monolayer TMDC from degradation and contamination [2]. It is widely assumed that $\mathrm{hBN}$ serves as a dielectric layer when forming a vertical heterostructure with the monolayer TMDC but the exciton coupling between the two is not well understood. Moreover, the effect of $\mathrm{hBN}$ on the luminescence properties of a TMDC heterostructure is not easy to probe as the optimal light absorption in TMDC and hBN occurs in two spectral ranges: near infrared and deep ultraviolet, respectively, due to distinct band gaps. Here, we present a correlative approach that combines cathodoluminescence (CL) and electron energy-loss spectroscopy (EELS) to investigate the electronic structure of monolayer WSe 2 stacked with $\mathrm{hBN}$ in various vertical heterostructure configurations.

We employed a focused electron beam to couple with excitons in monolayer $\mathrm{WSe}_{2}$ and $\mathrm{hBN}$, and measure the inter-band transitions using CL and EELS in an environmental scanning transmission electron microscope (ESTEM) equipped with a monochromated field-emission gun operated at $80 \mathrm{kV}$ (EELS energy resolution of $0.08 \mathrm{eV}$ ). A custom-built, free-space spectroscopy system is attached to the ESTEM column to collect the CL signal using a parabolic mirror positioned underneath the sample holder [3]. Three vertical heterostructures made of monolayer $\mathrm{WSe}_{2}$ and hBN (Fig. 1a) along with a freestanding monolayer WSe 2 are studied in this work. The heterostructures were processed using a nano-"squeegee" method using an atomic force microscopy (AFM) tip [4]. High-resolution TEM (HRTEM) of a $\mathrm{hBN} / \mathrm{WSe} 2 / \mathrm{hBN}$ heterostructure (Fig. 1b) shows the predominate lattice fringes of $\mathrm{hBN}$ and moiré fringes resulting from the small twisted alignment of the top and bottom hBN layers. Using Fourier-space filtering, we remove the real-space contrast (Fig. 1c) associated with the moire frequencies (green box, Fig. 1d) from the original HRTEM image and separate the $\{10-10\}$ lattice fringes between $\mathrm{hBN}$ (red circles $g_{n}$, Fig. 1d) and monolayer WSe2 (blue circles $g_{1}$, Fig. 1d). The filtered lattice fringes are shown in Figs. $1 \mathrm{e}$ and $\mathrm{f}$, respectively. EELS spectra at the low-loss region (Fig. 2a) indicates the hBN conduction band onset at $\approx 5.8 \mathrm{eV}$ for all of the three heterostructures. The inter-band transitions in $\mathrm{WSe}_{2}$ is shown in Fig. $2 \mathrm{~b}$ and compared to the spin-splitting intravalley transitions $\mathrm{A}$ and $\mathrm{B}$, reported to be at $\approx 1.69$ and $\approx$ $2.01 \mathrm{eV}$, respectively (vertical dashed lines, Fig. 2b) [5]. We found that the $\mathrm{hBN} / \mathrm{WSe} / \mathrm{hBN}$ displayed the most spectral redshift in both $\mathrm{A}$ and $\mathrm{B}$ excitons and peak broadening, indicating the indirect transitions that result from intervalley, momentum-forbidden dark excitons coupled through interlayer orbital hybridization due to the $\mathrm{hBN}$ encapsulation [6]. The $\mathrm{CL}$ intensity of $\mathrm{hBN} / \mathrm{WSe} / \mathrm{hBN}$ obtained at room temperature shows a large intensity enhancement and a shoulder peak at the lower energy side (Fig. 2c), 
both likely caused by the momentum-required transitions, when compared to the other spectra of the two heterostructures and the freestanding monolayer.

Our results show that correlative CL and EELS can provide insight to the optoelectronic properties of TMD heterostructures, including interlayer excitons coupling with wide-band-gap hBN. The multimodal spectroscopy and high-spatial-resolution imaging in an ESTEM will jumpstart the research on subgap localized excitons induced by point defects, strains or surface adsorbates.
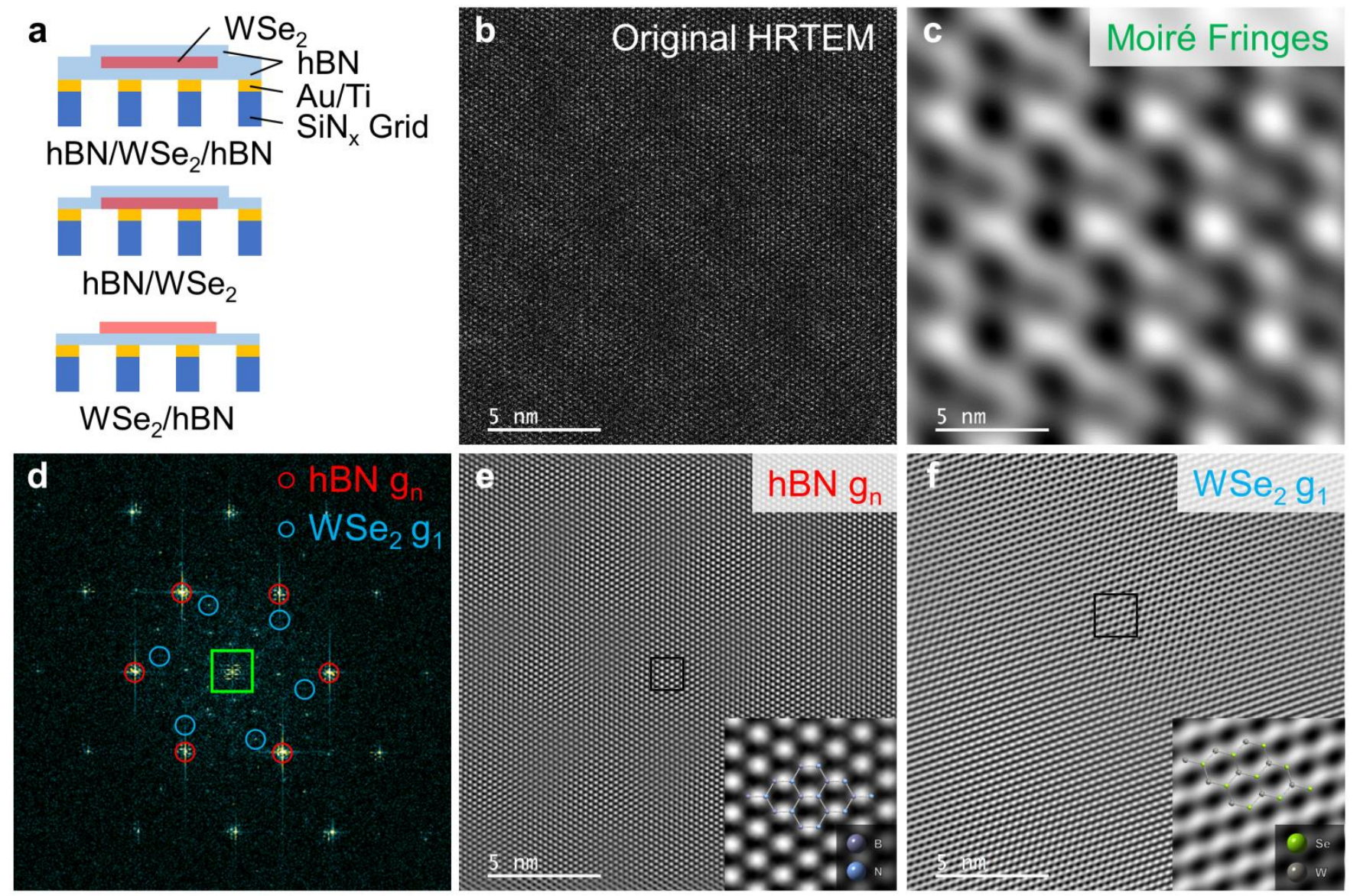

Figure 1. Figure 1. Image analysis for vertical heterostructures made of monolayer WSe2 and hBN. (a) Schematic of three vertical heterostructures on SiNx TEM grids coated with Au/Ti. (b) HRTEM micrograph of a hBN/WSe2/hBN heterostructure aligned to <0001> zone axis. (c) Fast Fourier transform (FFT) diffractogram of (b). (d) Real-space contrast of moire fringes obtained using inverse FFT of (c) after applying band-pass filters to each moiré frequencies displayed within the green box in (c). (e-f) Lattice fringes of hBN (e) and monolayer WSe2 (f) obtained using inverse FFT of (c) after applying bandpass filters to reflections of $\mathrm{hBN}$ gn (red circles) and WSe $2 \mathrm{~g} 1$ (blue circles) in (c), respectively. Insets show magnified images of the areas indicated by a black box and the overlaid atomic-structure model of hBN and WSe2, respectively. 

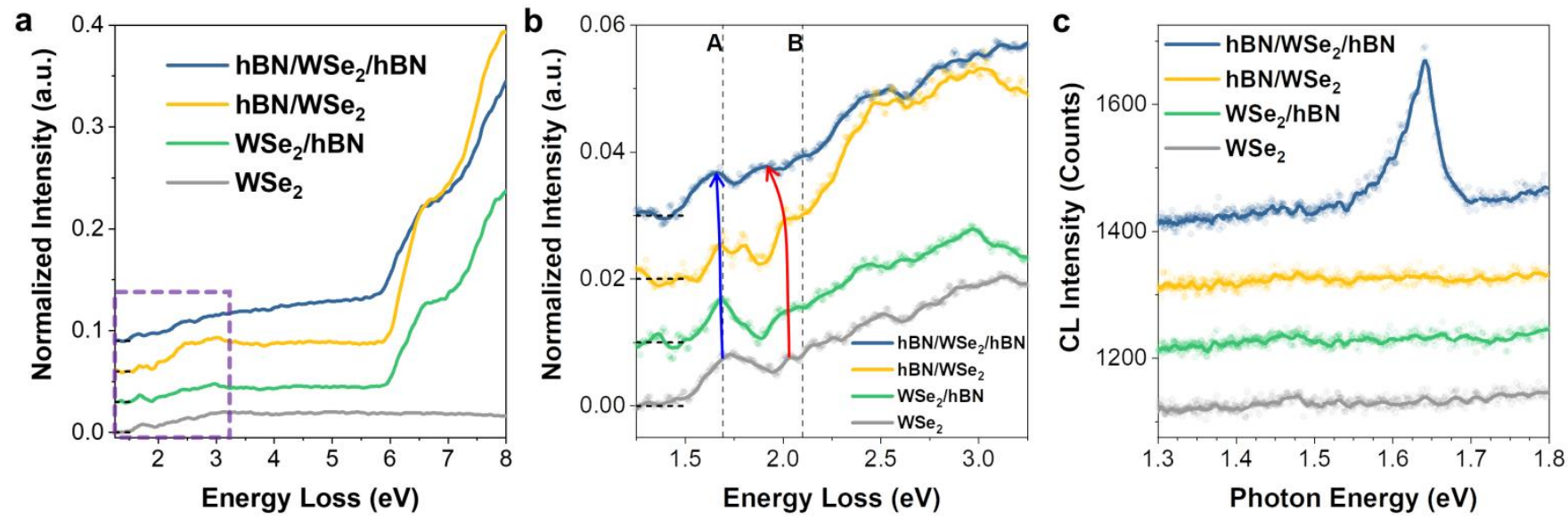

Figure 2. Figure 2. Correlative EELS and CL in STEM mode to probe the three vertical heterostructures and freestanding monolayer WSe2. (a) EELS spectra from $1.25 \mathrm{eV}$ to $8 \mathrm{eV}$ showing the conduction band onsets of both WSe 2 at $1.5 \mathrm{eV}$ and $\mathrm{hBN}$ at $\approx 5.8 \mathrm{eV}$. Spectra are shifted vertically for clarity and normalized to their relative zero-loss peak intensity. (b) EELS spectra representing the indicated spectral region in (a) and showing the spectra redshifts in the vertical heterostructures, indicated by the solid arrows. The vertical dashed lines indicating the spin-splitting intravalley excitons A and B previously reported at $\approx 1.69 \mathrm{eV}$ and $\approx 2.1 \mathrm{eV}$. (c) $\mathrm{CL}$ spectra showing a large intensity enhancement for the $\mathrm{hBN} / \mathrm{WSe} 2 / \mathrm{hBN}$, whereas no significant peak in the other three configurations observed at the identical scale.

References

[1] Q Wang et al., Nat. Nanotch. 7 (2012) p. 699.

[2] S Manzeli et al., Nat. Rev. Mater. 2 (2017) p. 17033.

[3] Y Yoon and WCD Yang et al., ACS Appl. Mater. Interfaces 11 (2019) p. 47037.

[4] MR Rosenberger et al., ACS Appl. Mater. Interfaces 10 (2018), p. 10379.

[5] J Hong et al. Phys. Rev. Lett. 124 (2020) p. 087401.

[6] Y Uchiyama et al. npj 2D Mater. Appl. 3 (2019) p. 26. 